\title{
Association between red blood cell distribution width and macrovascular and microvascular complications in diabetes
}

\author{
N. Malandrino $\cdot$ W. C. Wu $~$ T. H. Taveira • \\ H. B. Whitlatch • R. J. Smith
}

Received: 10 May 2011 / Accepted: 9 September 2011 / Published online: 15 October 2011

(C) Springer-Verlag 2011

\begin{abstract}
Aims/hypothesis Red blood cell distribution width (RDW) has been reported to be a risk marker of morbidity and mortality for cardiovascular disease in various study populations. However, no studies have investigated the relationship between RDW and diabetes complications. We
\end{abstract}

N. Malandrino $\cdot$ H. B. Whitlatch $\cdot$ R. J. Smith

Division of Endocrinology, Department of Medicine,

Warren Alpert Medical School of Brown University,

Providence, RI, USA

\section{N. Malandrino}

Department of Internal Medicine, Catholic University,

Rome, Italy

W. C. Wu $\cdot$ T. H. Taveira

Center on Systems, Outcomes and Quality in Chronic Disease and Rehabilitation, Research Enhancement Award Program,

Providence Veterans Affairs Medical Center,

Providence, RI, USA

W. C. Wu $\cdot$ T. H. Taveira

Division of Cardiology, Department of Medicine,

Warren Alpert Medical School of Brown University,

Providence, RI, USA

T. H. Taveira

University of Rhode Island College of Pharmacy,

Kingston, RI, USA

H. B. Whitlatch

Department of Medicine,

Providence Veterans Affairs Medical Center,

Providence, RI, USA

N. Malandrino $\cdot$ H. B. Whitlatch $\cdot$ R. J. Smith $(\bowtie)$

Hallett Center for Diabetes and Endocrinology,

900 Warren Avenue, Suite 300,

East Providence, RI 02914, USA

e-mail: Robert_J_Smith@Brown.edu therefore evaluated RDW as a marker of macrovascular and microvascular complications in a nationally representative sample of the adult diabetes population in the USA.

Methods A cross-sectional study was performed using the nationwide 1988 to 1994 data set from the Third National Health and Nutrition Examination Survey. The association between RDW quartiles and macrovascular and microvascular complications was evaluated in 2,497 non-pregnant adults aged 20 years and older and affected by diabetes. Logistic regression modelling was used to adjust for potential confounding.

Results Compared with the lowest RDW quartile, higher RDW values (3rd and 4th quartiles) were associated with increased adjusted odds of any vascular complication (OR 4th quartile 2.06 [95\% CI $1.11,3.83]$ ), myocardial infarction (OR 4th quartile 2.45 [95\% CI 1.13, 5.28]), heart failure (OR 4th quartile 4.40 [95\% CI 1.99, 9.72]), stroke (OR 4th quartile 2.56 [95\% CI 1.21, 5.42]) and nephropathy (OR 4th quartile 2.33 [95\% CI 1.42, 3.82]). The odds of developing diabetic retinopathy were not significantly increased across RDW quartiles.

Conclusions/interpretation Higher RDW values are associated with increased odds of developing cardiovascular disease and nephropathy in a nationally representative sample of USA adults with diabetes. RDW may be an important clinical marker of vascular complications in diabetes and one that is independent of traditional risk factors and disease duration.

Keywords Cardiovascular complications · Microvascular and macrovascular complications · Nephropathy .

NHANES $\cdot$ Red blood cell distribution width
Abbreviations
CRP
C-reactive protein
CVD
Cardiovascular disease 


$\begin{array}{ll}\text { IRB } & \text { Institutional Review Board } \\ \text { MCV } & \text { Mean corpuscular volume } \\ \text { NHANES III } & \text { Third National Health and Nutrition } \\ & \text { Examination Survey } \\ \text { RDW } & \text { Red blood cell distribution width } \\ \text { TSH } & \text { Thyroid-stimulating hormone } \\ \text { UACR } & \text { Urinary albumin/creatinine ratio }\end{array}$

\section{Introduction}

Diabetes mellitus is a chronic illness characterised by increased risk of macrovascular and microvascular complications. Cardiovascular disease (CVD) is the primary cause of mortality in patients with diabetes [1]. Microvascular complications are a major cause of morbidity in diabetes populations and have been associated with heightened risk of systemic vascular complications [1,2]. The identification of markers of macrovascular and microvascular disease could provide new information about the pathogenesis and early diagnosis of diabetes complications and facilitate decision-making in terms of prevention and treatment.

Red blood cell distribution width (RDW) is a measure of the variability in size of circulating erythrocytes (anisocytosis) [3] and is routinely assessed in the evaluation of anaemia. Increased RDW levels are related to impaired erythropoiesis or erythrocyte degradation [3].

RDW levels, even within the normal range, have been strongly and independently associated with risk of all-cause and cardiovascular mortality in patients with CVD $[4,5]$ and in the general population $[6,7]$. RDW has also been related to increased risk of new cardiovascular events in people with previous myocardial infarction [5]. However, no studies have investigated the relationship between RDW and macrovascular complications in a selected diabetes population. Furthermore, no data on the relationship between RDW and diabetic microvascular complications have been reported.

We therefore sought to evaluate RDW as a marker of vascular complications in a nationally representative sample of the adult diabetes population in the USA and to evaluate potential differences in the association between RDW and macrovascular and microvascular complications. The study's primary outcome was the presence, across RDW quartiles, of any of the following vascular complications: myocardial infarction, heart failure, stroke, retinopathy and nephropathy. The secondary outcome was the presence of individual vascular complications across RDW quartiles.

\section{Methods}

Study overview A cross-sectional design was used to investigate the relationship between RDW and macro- vascular (myocardial infarction, heart failure, stroke) and microvascular (retinopathy, nephropathy) complications in participants who were aged 20 years and older, and had diabetes. Participant data were from the Third National Health and Nutrition Examination Survey (NHANES III), 1988 to 1994 database. NHANES III was developed to provide health status information on a nationally representative sample of the civilian non-institutionalised population of the USA. The survey consisted of an in-home interview, in which participants were queried about health behaviours, health status and risk factors, and a physical examination and laboratory tests were conducted at a mobile examination centre [8]. The NHANES protocol was approved by the National Center for Health Statistics Institutional Review Board (IRB) and written informed consent for data collection was obtained from all survey participants [9]. The present protocol was reviewed by the IRBs of Rhode Island Hospital and Providence Veterans Affairs Medical Center. Since the NHANES data were already de-identified [9], both IRBs considered the study exempt from full board review.

Of the 18,825 NHANES III participants aged 20 years and older, 17,030 had blood samples taken. Among these, 332 participants were pregnant and excluded. Of the remaining 16,698 participants, 2,756 had diabetes. RDW values were available for 2,497 of these, and they constituted our study population.

Demographic and social characteristics Information on age, race/ethnicity, education, smoking and alcohol consumption was collected using a standardised questionnaire. Smoking was categorised as current, past or never. Past smokers were those who reported having smoked at least 100 cigarettes during their lifetime, but did not currently smoke cigarettes. Never smokers were those who said they had smoked less than 100 cigarettes during their life. Moderate drinkers were defined as men who had consumed one to two servings of alcohol per day or women who had consumed at least one serving of alcohol per day during the past month. Never drinkers were those who had not drunk any alcoholic beverage during the past month. No participants reported more than moderate drinking in our sample.

Laboratory procedures Each participant had a blood specimen drawn from an antecubital vein. A device (S-plus Jr Counter; Coulter Electronics, Hialeah, FL, USA) was used to measure RDW, haemoglobin and mean corpuscular volume (MCV). The maximum acceptable coefficient of variation for RDW was $3.2 \%$.

Plasma glucose, $\mathrm{HbA}_{1 \mathrm{c}}$, serum lipids, C-reactive protein (CRP), thyroid-stimulating hormone (TSH), serum and urinary albumin and creatinine, iron and vitamin levels 
were analysed by standardised laboratory methods, as previously reported [8].

Medical history and examination Diabetes mellitus was defined as fasting plasma glucose $\geq 7.0 \mathrm{mmol} / 1$ and/or 2 -h postload plasma glucose $\geq 11.1 \mathrm{mmol} / 1$ (both determined after overnight fasting for at least $8 \mathrm{~h}$ ) and/or $\mathrm{HbA}_{1 \mathrm{c}} \geq 6.5 \%$ (48 $\mathrm{mmol} / \mathrm{mol}$ ) and/or self-reported diabetes medication use and/or self-reported medical diagnosis of diabetes (other than gestational diabetes) at the time of the interview.

History of heart attack, congestive heart failure, stroke and pulmonary disease (asthma, chronic bronchitis, emphysema) were defined on the basis of self-reported medical diagnosis.

The analysis of diabetic retinopathy was restricted to adults aged 40 years or older who underwent fundus photography according to the NHANES study protocol. Photographic fields were graded, in a masked fashion, by trained graders at the Ocular Epidemiology Grading Center, University of Wisconsin Medical School. The presence of retinopathy was classified using the Modified Airlie House Classification scheme [10]. A summary diabetic retinopathy score was then calculated. Participants without lesions indicative of diabetic retinopathy, or with hard or soft exudates, intraretinal microvascular abnormalities or haemorrhages without microaneurysms, or with non-diabetic retinopathy were considered to be not affected by diabetic retinopathy. Mild diabetic retinopathy was defined as the presence of only microaneurysms or early non-proliferative diabetic retinopathy. Moderate diabetic retinopathy was defined as the presence of moderate or severe nonproliferative diabetic retinopathy. Proliferative diabetic retinopathy was defined by the presence of at least one of the following two conditions: (1) fibrous proliferation only or proliferative diabetic retinopathy that meets standard high-risk characteristics for severe visual loss; and/or (2) no diabetic retinopathy or any level of diabetic retinopathy on examination, but evidence of photocoagulation treatment outside retinal vascular arcades [11].

To assess renal function, creatinine values analysed in NHANES III were recalibrated to the Cleveland Clinic Research Laboratory standard by applying the following formula: standardised creatinine $=-0.184+(0.960 \times$ uncalibrated serum creatinine in $\mu \mathrm{mol} / \mathrm{l})$ [12]. Estimated GFR was then calculated using the following Modification of Diet in Renal Disease formula: estimated GFR $=175 \times($ standardised creatinine / 88.4 $)^{-1.154} \times(\mathrm{age})^{-0.203} \times(0.742$ if the participant is female $) \times(1.212$ if the participant is black $) \times 0.0167$. Urinary albumin/creatinine ratio (UACR) was calculated by dividing the urinary albumin value by the urinary creatinine concentration. Diabetic nephropathy was defined as follows: stage $1 \mathrm{UACR} \geq 3.4 \mathrm{mg} / \mathrm{mmol}$ with an estimated GFR $\geq 1.5 \mathrm{ml} \mathrm{s}^{-1} \mathrm{~m}^{-2}$; stage 2 UACR $\geq 3.4 \mathrm{mg} / \mathrm{mmol}$ with an estimated GFR of 1 to $1.49 \mathrm{ml} \mathrm{s}^{-1} \mathrm{~m}^{-2}$; stage 3 estimated GFR of 0.5 to $0.99 \mathrm{ml} \mathrm{s}^{-1} \mathrm{~m}^{-2}$; stage 4 estimated GFR of 0.25 to $0.49 \mathrm{ml} \mathrm{s}^{-1} \mathrm{~m}^{-2}$; and stage 5 estimated GFR $<0.25 \mathrm{ml} \mathrm{s}^{-1} \mathrm{~m}^{-2}$ [13].

Participants were included in the 'any vascular complication' sample if they answered 'yes' to at least one question about diabetic complications, or were affected by retinopathy or nephropathy, even if data were missing for some complications. Participants who answered 'no' to all questions about diabetic complications were included in the group defined as 'not affected by any complication' ( $n=$ 876). Participants who answered 'no' to some questions and for whom data on the other questions were missing were excluded because it was not known whether or not they had complications $(n=374)$. Because of missing data, the number of participants in each secondary outcome sample was lower than 2,497 .

Information on recent treatment for anaemia (including blood transfusions) or acute illness (i.e. colds, flu, diarrhoea, vomiting, pneumonia or ear infections within the past 4 weeks), duration of diabetes and diabetes-related medication use was collected using a standardised questionnaire.

Iron deficiency was defined by at least two of the following three criteria: transferrin saturation $<15 \%$ (transferrin saturation is calculated as: iron/total iron binding capacity $\times 100$ ), serum ferritin level $<26.9 \mathrm{pmol} / 1$ and erythrocyte protoporphyrin level $>1.24 \mu \mathrm{mol} / 1$ [14]. Folate and vitamin $\mathrm{B}_{12}$ deficiencies were defined by erythrocyte folate levels $<232.49 \mathrm{nmol} / 1$ and serum vitamin $B_{12}$ levels $<147.6 \mathrm{pmol} / \mathrm{l}$, respectively [6].

Body mass index was calculated as weight in $\mathrm{kg}$ divided by height in $\mathrm{m}^{2}$. Systolic and diastolic BP readings were obtained after the participant had been seated quietly for $5 \mathrm{~min}$. Three to four consecutive measurements were taken on the same arm using a mercury sphygmomanometer, and the average value was considered for the analysis. Hypertension was defined on the basis of selfreported medical diagnosis of hypertension and/or, on examination, systolic BP of $\geq 130 \mathrm{mmHg}$ and/or diastolic $\mathrm{BP}$ of $\geq 80 \mathrm{mmHg}$ [1].

Statistical analysis The RDW values ranged from $11.1 \%$ to $24.1 \%$ (median $13.15 \%$; interquartile range $12.6-13.85 \%$ ). To evaluate the association between RDW and clinical outcomes, RDW values were examined as a continuous variable as well as divided into quartiles using the following cut-offs: 11.1 to $12.6 \%,>12.6$ to $<13.2 \%, 13.2$ to $<13.9 \%, 13.9$ to $24.1 \%$. Participant characteristics were studied across quartiles of RDW, and differences between groups were examined by simple linear regression for continuous variables and by $\chi^{2}$ test for categorical variables. Skewed variables were logarithmically transformed to obtain a normal distribution. 
The association between clinical outcomes and covariates was examined with logistic regression models and expressed as OR $(95 \% \mathrm{CI})$. Using the first quartile of RDW as reference group, covariates that modified the relationship between RDW and the presence of any combination of vascular complications (primary study outcome) by $>10 \%$, i.e. age, CRP and $\mathrm{HbA}_{1 \mathrm{c}}$, were considered as potential confounders and hence included in the final regression model. Other variables, such as alcohol consumption, HDLcholesterol, triacylglycerol, TSH, history of acute illness or pulmonary disease, and diabetes treatment were also tested and did not significantly modify the relationship between RDW and the primary outcome. Moreover, on the basis of previous literature [7], additional variables independently associated with diabetic complications or with RDW were selected as covariates in the fully adjusted model, namely: sex, race/ethnicity, education, hypertension, smoking, BMI, serum cholesterol levels, duration of diabetes, haemoglobin, $\mathrm{MCV}$, iron, vitamin $\mathrm{B}_{12}$ and folate deficiency, and hypoalbuminaemia.

Multiple imputation was used to impute missing values for education, hypertension, BMI, total cholesterol, HDLcholesterol, triacylglycerol, CRP, TSH, albumin, vitamin $\mathrm{B}_{12}$ and folate deficiency, $\mathrm{HbA}_{1 \mathrm{c}}$, recent treatment for anaemia or acute illness, duration of diabetes and diabetes treatment. Multiple imputation is an efficient method for imputing missing values when it is reasonable to assume that data are missing at random. Variables predictive of the items being imputed and variables that were used in subsequent logistic analyses of the multiple imputed data were added as predictors in the model for multiple imputation. Five imputed datasets were generated and logistic analyses of the multiple-imputed data were performed.

Analyses of statistical interactions were performed by adding, in the final adjusted model, the interaction terms obtained from combining RDW with each of the following variables: age, haemoglobin, $\mathrm{MCV}, \mathrm{BMI}, \mathrm{CRP}, \mathrm{HbA}_{1 \mathrm{c}}$, smoking and hypertension. When a value of $p<0.05$ was observed for the two variables and the interaction term, the relationship between RDW and primary outcome was evaluated by subgroups.

We also performed three subgroup analyses. In the first analysis, we excluded individuals recently treated for anaemia. In the second analysis, we excluded individuals with RDW values outside the normal range (11.8-14.8\%). Finally, considering that vitamin $\mathrm{B}_{12}$ levels were determined only in phase 2 of NHANES III (1991-1994), a subgroup analysis excluding participants with missing vitamin $\mathrm{B}_{12}$ values was performed. In all the subgroups, we analysed the relationship between RDW and vascular complications, after adjusting for confounders by using the multiple variable model reported above.
Also, a sensitivity analysis was conducted to compare the estimates obtained from imputed data with those observed in complete case analysis.

Because the NHANES uses a complex multistage probability sampling design to provide estimates for the USA population, all analyses were performed using the designated weighting specified in the NHANES III database. The total NHANES III pseudostratum was used as strata variable, the total NHANES III pseudoprimary sampling units as survey sampling units and the final weight of the total mobile exam centre-examined sample as sampling unit weight. Sampling weights accounted for the non-random sampling, including oversampling of the elderly, non-Hispanic blacks, and Mexican-Americans, and also for non-response, by adjusting for different probabilities of selection.

Statistical analyses were performed using STATA SE 11.1 (StataCorp, College Station, TX, USA). A value of $p<0.05$ was considered significant.

\section{Results}

In our population $(n=2,497)$, the diagnosis of diabetes was based on self-report in 363 participants (14.5\%), fasting hyperglycaemia in 87 participants $(3.5 \%)$, postprandial hyperglycaemia in 509 participants $(20.4 \%)$ and $\mathrm{HbA}_{1 \mathrm{c}}$ $\geq 6.5 \%(48 \mathrm{mmol} / \mathrm{mol})$ in 214 participants $(8.6 \%)$. The rest of the sample $(n=1,324,53 \%)$ had more than one criterion for diagnosis of diabetes. Notably, nearly half of the participants $(n=1,149,46 \%)$ were not aware of their diagnosis of diabetes.

Table 1 lists baseline participant characteristics for the entire sample, stratified according to RDW quartiles. Participants with higher RDW levels were more likely to be older, less educated and currently smoking. The percentage of non-Hispanic blacks, and of those with hypertension and severe nephropathy, as well as BMI, CRP and UACR levels increased with increasing RDW quartiles. In addition, haemoglobin levels and estimated GFR decreased with increasing RDW quartiles. Despite significant differences in $\mathrm{HbA}_{1 \mathrm{c}}$ levels between RDW quartiles, this relationship did not follow a linear trend. Finally, participants with higher RDW values had a higher prevalence of nutritional deficiencies (in particular iron, folate and albumin deficiency). Interestingly, these significant relationships persisted even after excluding participants with vascular complications.

Multivariate logistic regression analysis (Table 2) showed RDW quartiles, age, smoking, hypertension, BMI, $\mathrm{CRP}$ and $\mathrm{HbA}_{1 \mathrm{c}}$ to be variables independently associated with the presence of macrovascular and/or microvascular complications. After adjustment for potential confounding 
Table 1 Baseline participant characteristics across RDW quartiles

\begin{tabular}{|c|c|c|c|c|c|}
\hline \multirow[t]{2}{*}{ Variable } & \multicolumn{4}{|l|}{ Quartiles } & \multirow[t]{2}{*}{$p$ value } \\
\hline & $1 \mathrm{st}$ & 2nd & $3 \mathrm{rd}$ & 4th & \\
\hline$n$ & 631 & 656 & 593 & 617 & \\
\hline \multicolumn{6}{|l|}{ RDW } \\
\hline Unweighted (median) & 12.3 & 12.9 & 13.45 & 14.6 & \\
\hline Interquartile range & $11.1-12.6$ & $>12.6$ to $<13.2$ & 13.2 to $<13.9$ & $13.9-24.1$ & \\
\hline Age (years) & $56.5 \pm 12.5$ & $59.4 \pm 12.4$ & $60.7 \pm 14.1$ & $62.6 \pm 15.7$ & $<0.001$ \\
\hline Female sex $(\%)$ & 57.4 & 49.8 & 55.8 & 59.5 & 0.1 \\
\hline Ethnicity $(\%)$ & & & & & $<0.001$ \\
\hline Non-Hispanic white & 78.9 & 76.8 & 72.4 & 61.9 & \\
\hline Non-Hispanic black & 8.0 & 9.0 & 14.8 & 26.1 & \\
\hline Mexican-American & 6.0 & 5.7 & 4.9 & 6.3 & \\
\hline Other $^{\mathrm{a}}$ & 7.1 & 8.5 & 7.9 & 5.7 & \\
\hline Graduated high school (\%) & & & & & $<0.001$ \\
\hline Yes & 67.4 & 62.7 & 56.7 & 46.1 & \\
\hline No & 32.5 & 37.3 & 42.5 & 53.7 & \\
\hline Unknown & 0.1 & - & 0.8 & 0.2 & \\
\hline Smoking (\%) & & & & & 0.009 \\
\hline Never & 45.6 & 38.5 & 39.8 & 38.0 & \\
\hline Former & 39.0 & 44.2 & 33.8 & 38.2 & \\
\hline Current & 15.4 & 17.3 & 26.4 & 23.8 & \\
\hline Hypertension (\%) & & & & & 0.06 \\
\hline Yes & 72.8 & 74.5 & 78.5 & 84.2 & \\
\hline No & 27.0 & 25.0 & 21.5 & 15.7 & \\
\hline Unknown & 0.2 & 0.5 & - & 0.1 & \\
\hline BMI $\left(\mathrm{kg} / \mathrm{m}^{2}\right)$ & $28.8 \pm 5.2$ & $29.0 \pm 5.7$ & $30.2 \pm 7.0$ & $31.0 \pm 8.9$ & $<0.001$ \\
\hline Unknown (\%) & 0.1 & 0.3 & 0.2 & - & \\
\hline Total cholesterol (mmol/l) & $5.81 \pm 1.15$ & $5.76 \pm 1.05$ & $5.81 \pm 1.33$ & $5.73 \pm 1.7$ & 0.78 \\
\hline Unknown (\%) & 0.8 & 0.4 & 1.8 & 2.7 & \\
\hline CRP (nmol/l) & $49.5 \pm 66.7$ & $48.6 \pm 66.7$ & $66.7 \pm 85.7$ & $100.0 \pm 138.1$ & $<0.001$ \\
\hline Unknown (\%) & 1.1 & 1.5 & 3.1 & 3.1 & \\
\hline Haemoglobin (g/l) & $145 \pm 11$ & $143 \pm 12$ & $142 \pm 15$ & $134 \pm 19$ & $<0.001$ \\
\hline MCV (fl) & $90.7 \pm 3.4$ & $90.3 \pm 4.1$ & $89.0 \pm 5.7$ & $88.4 \pm 9.6$ & $<0.001$ \\
\hline Serum albumin (g/l) & $41.6 \pm 3.0$ & $41.0 \pm 3.0$ & $40.0 \pm 3.0$ & $39.0 \pm 5.0$ & $<0.001$ \\
\hline Unknown (\%) & 1.4 & 1.3 & 3.9 & 3.2 & \\
\hline Iron deficiency $(\%)$ & & & & & $<0.001$ \\
\hline Yes & 0.8 & 2.3 & 10.6 & 21.2 & \\
\hline No & 99.2 & 97.7 & 89.4 & 78.8 & \\
\hline Vitamin $\mathrm{B}_{12}$ deficiency ${ }^{\mathrm{b}}(\%)$ & & & & & 0.57 \\
\hline Yes & 0.4 & 1.5 & 1.4 & 1.9 & \\
\hline No & 49.0 & 52.5 & 53.5 & 53.5 & \\
\hline Unknown & 50.6 & 46.0 & 45.1 & 44.6 & \\
\hline Folate deficiency (\%) & & & & & $<0.001$ \\
\hline Yes & 2.3 & 4.9 & 8.7 & 7.8 & \\
\hline No & 97.5 & 94.8 & 91.0 & 90.6 & \\
\hline Unknown & 0.2 & 0.3 & 0.3 & 1.6 & \\
\hline $\mathrm{HbA}_{1 \mathrm{c}}$ & & & & & 0.03 \\
\hline$\%$ & $7.0 \pm 1.9$ & $6.8 \pm 1.7$ & $7.2 \pm 2.1$ & $6.7 \pm 1.9$ & \\
\hline $\mathrm{mmol} / \mathrm{mol}$ & 53.0 & 51.0 & 55.0 & 50.0 & \\
\hline Unknown (\%) & 0.6 & - & 0.1 & - & \\
\hline
\end{tabular}


Table 1 (continued)

\begin{tabular}{|c|c|c|c|c|c|}
\hline \multirow[t]{2}{*}{ Variable } & \multicolumn{4}{|l|}{ Quartiles } & \multirow[t]{2}{*}{$p$ value } \\
\hline & $1 \mathrm{st}$ & 2 nd & $3 \mathrm{rd}$ & 4th & \\
\hline Diabetes duration (years) & $9.1 \pm 8.2$ & $9.2 \pm 9.4$ & $9.9 \pm 9.0$ & $10.2 \pm 10.2$ & 0.88 \\
\hline Unknown (\%) & 57.7 & 56.2 & 50.3 & 52.7 & \\
\hline Estimated GFR $\left(\mathrm{ml} \mathrm{s}^{-1} \mathrm{~m}^{-2}\right)$ & $1.48 \pm 0.37$ & $1.41 \pm 0.37$ & $1.37 \pm 0.41$ & $1.29 \pm 0.59$ & $<0.001$ \\
\hline Unknown (\%) & 1.4 & 1.3 & 3.9 & 3.2 & \\
\hline UACR (mg/mmol) & $7.65 \pm 37.3$ & $7.94 \pm 43.6$ & $10.3 \pm 43.2$ & $21.8 \pm 98.3$ & 0.001 \\
\hline Unknown (\%) & 2.0 & 1.0 & 3.4 & 7.2 & \\
\hline Nephropathy stages (\%) & & & & & $<0.001$ \\
\hline No nephropathy & 70.5 & 66.7 & 59.7 & 46.3 & \\
\hline Stage 1 & 7.3 & 5.7 & 8.1 & 7.5 & \\
\hline Stage 2 & 11.0 & 12.9 & 9.9 & 11.3 & \\
\hline Stage 3 & 8.6 & 12.1 & 15.0 & 25.3 & \\
\hline Stages $4-5$ & - & 0.4 & 0.5 & 3.6 & \\
\hline Unknown & 2.6 & 2.2 & 6.8 & 6.0 & \\
\hline
\end{tabular}

Unless otherwise indicated, data are weighted means \pm SD

${ }^{a}$ Race other than white, black or Mexican-American

${ }^{b}$ Vitamin $B_{12}$ levels were determined only in phase 2 of NHANES III (1991 to 1994)

Table 2 Univariate and multivariate predictors ${ }^{\mathrm{a}}$ of 'any vascular complication, ${ }^{\text {, }}$ across RDW quartiles

\footnotetext{
${ }^{a}$ Variables included in the final adjusted model: age, sex, race/ ethnicity, education, smoking, hypertension, BMI, total cholesterol levels, CRP, haemoglobin, MCV, albumin levels, iron deficiency, vitamin $\mathrm{B}_{12}$ deficiency, folate deficiency, $\mathrm{HbA}_{1 \mathrm{c}}$ and duration of diabetes ${ }^{\mathrm{b}}$ Indicates the presence of at least one of the following diabetic complications: myocardial infarction, heart failure, stroke, retinopathy and nephropathy

${ }^{\mathrm{c}}$ Race other than white, black or Mexican-American

${ }^{*} p<0.05$
}

\begin{tabular}{lll}
\hline Variable & Crude OR $(95 \% \mathrm{CI})$ & Adjusted OR $(95 \% \mathrm{CI})$ \\
\hline RDW 2nd quartile vs RDW 1st quartile & $1.31(0.88,1.95)$ & $1.34(0.86,2.08)$ \\
RDW 3rd quartile vs RDW 1st quartile & $2.04(1.36,3.04)^{*}$ & $1.61(1.04,2.52)^{*}$ \\
RDW 4th quartile vs RDW 1st quartile & $2.76(1.74,4.36)^{*}$ & $2.06(1.11,3.83)^{*}$ \\
Age & $1.04(1.02,1.05)^{*}$ & $1.04(1.03,1.06)^{*}$ \\
Female vs male & $0.99(0.75,1.30)$ & $0.85(0.57,1.26)$ \\
Black vs white & $1.27(1.02,1.59)^{*}$ & $0.80(0.55,1.17)$ \\
Mexican-American vs white & $0.83(0.62,1.10)$ & $0.87(0.58,1.32)$ \\
Other races ${ }^{\mathrm{c}}$ vs white & $0.71(0.36,1.39)$ & $0.89(0.43,1.84)$ \\
High school vs no high school graduation & $0.73(0.55,0.97)^{*}$ & $0.92(0.68,1.25)$ \\
Former smoker vs never & $1.23(0.92,1.64)$ & $1.12(0.84,1.49)$ \\
Current smoker vs never & $1.08(0.71,1.65)$ & $1.68(1.01,2.78)^{*}$ \\
Hypertension vs no hypertension & $2.83(2.00,4.01)^{*}$ & $2.26(1.55,3.29)^{*}$ \\
BMI & $1.04(1.01,1.07)^{*}$ & $1.04(1.00,1.07)^{*}$ \\
Total cholesterol & $1.00(1.00,1.01)^{*}$ & $1.00(0.99,1.01)$ \\
CRP & $1.65(1.33,2.04)^{*}$ & $1.39(1.13,1.71)^{*}$ \\
Haemoglobin & $0.97(0.89,1.05)$ & $0.98(0.84,1.13)$ \\
MCV & $0.97(0.95,0.99)^{*}$ & $0.99(0.96,1.02)$ \\
Serum albumin & $0.55(0.38,0.80)^{*}$ & $1.07(0.70,1.63)$ \\
Iron deficiency vs no iron deficiency & $1.38(0.84,2.27)$ & $0.89(0.47,1.68)$ \\
Vitamin $B_{12}$ deficiency vs no B ${ }_{12}$ deficiency & $0.79(0.27,2.37)$ & $0.57(0.22,1.51)$ \\
Folate deficiency vs no folate deficiency & $0.68(0.39,1.16)$ & $0.57(0.32,1.01)$ \\
HbA ${ }_{1 \mathrm{c}}$ & $1.38(1.24,1.53)^{*}$ & $1.38(1.25,1.52)^{*}$ \\
Duration of diabetes & $1.03(1.01,1.05)^{*}$ & $1.02(0.99,1.05)$ \\
\hline & &
\end{tabular}


factors (Table 3), individuals in the highest quartiles of RDW were more likely than individuals in the lowest RDW quartile to have at least one diabetic complication. The same results were observed for myocardial infarction, heart failure, stroke and nephropathy. By contrast, the odds of having retinopathy were not significantly increased across RDW quartiles (Table 3).

Examining RDW as a continuous variable, the odds of having at least one diabetic complication increased by $21 \%$ for every $1 \%$ increment in RDW, adjusting for potential confounders (OR 1.21 [95\% CI 1.01, 1.45]).

Excluding individuals recently treated for anaemia did not significantly change the association between RDW and the primary outcome (Table 4). Subgroup analyses excluding participants with RDW levels outside the normal range or those with missing vitamin $\mathrm{B}_{12}$ values resulted in a smaller sample size and different magnitude of estimates. However, both subgroup analyses showed the same direction of estimates compared with the entire population (Table 4). Also, as shown in Fig. 1, the percentage of participants with at least one diabetic complication significantly increased across RDW quartiles in the entire diabetic population and in the three subgroup analyses. These findings suggest that the association between RDW and the primary outcome is valid for all RDW values, is not just driven by abnormal RDW values alone and is also independent of vitamin $\mathrm{B}_{12}$ deficiency.

Sensitivity analysis conducted with and without the imputed data in the model yielded similar results. The estimates with complete case analysis $(n=581)$ showed the same direction as those with imputed data (ORs for primary outcome 2nd quartile 1.41 [95\% CI 0.55, 3.64], 3rd quartile 2.69 [95\% CI $0.87,8.33]$ and 4 th quartile 3.40 [95\% CI $0.94,12.2])$.
Finally, analysis by subgroups stratified by CRP levels $(\leq 31.4 \mathrm{nmol} / 1$ and $>31.4 \mathrm{nmol} / \mathrm{l})$ showed the effect estimates to be similar with adjusted ORs ranging from 1.40 to $2.32(95 \%$ CI $0.77,2.55$ to $1.07,5.00)$ and 1.11 to $2.21(95 \%$ CI $0.65,1.90$ to $1.09,4.48)$, respectively for the different quartiles of RDW levels. This suggests that the association between RDW and any complication, as noted above (Statistical analysis), is attenuated by CRP in the entire sample and not just driven by a specific CRP subgroup.

\section{Discussion}

Red blood cell distribution width is a widely available and inexpensive test routinely performed as part of the complete blood cell count. Although higher RDW levels have been related to cardiovascular morbidity and mortality in several study populations [4-7], RDW has not been studied as a marker of complications in diabetes. In the present investigation, we used the NHANES III database to demonstrate a relationship between RDW and CVD in a selected diabetes population that is representative of approximately 18 million USA adults aged 20 years and older. This study also represents the first examination of the role of RDW as marker of two diabetic complications with a significant microvascular component, i.e. retinopathy and nephropathy. We found nephropathy to be significantly associated with RDW, but no relationship was found between retinopathy and RDW levels.

Increased RDW levels can be a consequence of anaemia or anaemia-related nutritional deficiencies. However, the association between clinical outcomes and RDW persisted after adjusting for multiple potential confounding factors, including haemoglobin, iron, vitamin $\mathrm{B}_{12}$ and folate

Table 3 Adjusted OR for each diabetes complication ${ }^{\mathrm{a}}$ across RDW quartiles

\begin{tabular}{|c|c|c|c|c|c|c|}
\hline \multicolumn{3}{|c|}{ Participants and events per complication } & \multicolumn{4}{|c|}{ RDW quartiles, unweighted median (interquartile range) } \\
\hline Complication & $\begin{array}{l}\text { Participants }(n) \text {, } \\
\text { unweighted }\end{array}$ & $\begin{array}{l}\text { Events, } n(\%) \\
\text { unweighted }\end{array}$ & $\begin{array}{l}1 \mathrm{st}, 12.3 \\
(11.1-12.6)\end{array}$ & $\begin{array}{l}\text { 2nd, } 12.9 \\
(>12.6 \text { to }<13.2)\end{array}$ & $\begin{array}{l}\text { 3rd, } 13.45 \\
(13.2 \text { to }<13.9)\end{array}$ & $\begin{array}{l}\text { 4th, } 14.6 \\
(13.9-24.1)\end{array}$ \\
\hline Any vascular complication & 2,123 & $1,247(58.7)$ & Reference & $1.34(0.86,2.08)$ & $1.61(1.04,2.52)^{*}$ & $2.06(1.11,3.83)^{*}$ \\
\hline Myocardial infarction & 2,464 & $268(10.9)$ & & $1.70(0.95,3.06)$ & $2.45(1.34,4.47)^{*}$ & $2.45(1.13,5.28)^{*}$ \\
\hline Heart failure & 2,490 & $251(10)$ & & $2.39(1.16,4.98)^{*}$ & $2.39(1.18,4.86)^{*}$ & $4.40(1.99,9.72)^{*}$ \\
\hline Stroke & 2,494 & $193(7.7)$ & & $2.04(1.07,3.91)^{*}$ & $2.78(1.36,5.68)^{*}$ & $2.56(1.21,5.42)^{*}$ \\
\hline Retinopathy & 1,832 & $270(14.7)$ & & $0.69(0.39,1.22)$ & $1.09(0.61,1.97)$ & $1.06(0.37,3.03)$ \\
\hline Nephropathy & 2,345 & $911(38.8)$ & & $1.28(0.81,2.02)$ & $1.20(0.82,1.76)$ & $2.33(1.42,3.82)^{*}$ \\
\hline
\end{tabular}

${ }^{\mathrm{a}}$ Adjusted for age, sex, race/ethnicity, education, smoking, BMI, hypertension, total cholesterol levels, CRP, HbA $1 \mathrm{c}$, duration of diabetes, haemoglobin, $\mathrm{MCV}$, iron deficiency, vitamin $\mathrm{B}_{12}$ deficiency, folate deficiency and albumin levels

$* p<0.05$ 
Table 4 Adjusted OR for 'any vascular complication' ${ }^{\text {a }}$ across RDW quartiles in the subgroup analyses ${ }^{\mathrm{b}}$

\begin{tabular}{|c|c|c|c|c|c|c|}
\hline \multicolumn{3}{|c|}{ Participants and events per complication } & \multicolumn{4}{|c|}{ RDW quartiles } \\
\hline Complication & $\begin{array}{l}\text { Participants }(n) \text {, } \\
\text { unweighted }\end{array}$ & $\begin{array}{l}\text { Events }(n) \text {, } \\
\text { unweighted }\end{array}$ & $1 \mathrm{st}$ & 2 nd & $3 \mathrm{rd}$ & 4 th \\
\hline Any vascular complication ${ }^{\mathrm{c}}$ & 1,956 & 1,116 & Reference & $1.31(0.83,2.05)$ & $1.64(1.05,2.54)^{*}$ & $1.97(1.07,3.63)^{*}$ \\
\hline Any vascular complication ${ }^{\mathrm{d}}$ & 1,866 & 1,064 & & $1.31(0.84,2.03)$ & $1.38(0.92,2.09)$ & $1.82(0.99,3.35)^{*}$ \\
\hline Any vascular complication ${ }^{\mathrm{e}}$ & 1,113 & 650 & & $1.19(0.78,1.83)$ & $1.60(0.81,3.19)$ & $2.24(0.84,5.95)$ \\
\hline
\end{tabular}

${ }^{\mathrm{a}}$ Indicates the presence of at least one of the following diabetic complications: myocardial infarction, heart failure, stroke, retinopathy and nephropathy

${ }^{\mathrm{b}}$ Adjusted for age, sex, race/ethnicity, education, smoking, BMI, hypertension, total cholesterol levels, CRP, HbA $1 \mathrm{c}$, duration of diabetes, haemoglobin, $\mathrm{MCV}$, iron deficiency, vitamin $\mathrm{B}_{12}$ deficiency, folate deficiency and albumin levels

${ }^{\mathrm{c}}$ Excluding participants recently treated for anaemia

${ }^{\mathrm{d}}$ Excluding RDW values outside the normal range

${ }^{\mathrm{e}}$ Excluding participants with missing vitamin $\mathrm{B}_{12}$ values

$* p<0.05$

deficiency. Thus, the relationship between the clinical outcomes assessed and RDW is independent of anaemia and anaemia-related nutritional factors.

Inflammation may influence erythropoiesis, erythrocyte circulatory half-life and erythrocyte deformability, promoting anisocytosis and thus increasing RDW levels [15]. In a large unselected outpatient population, greater RDW values were independently associated with greater CRP levels [16], a well-established marker of inflammation and CVD [17]. Diabetes mellitus and the related metabolic syndrome are associated with chronic inflammation [18]. Heightened inflammatory activity has been demonstrated in apparently
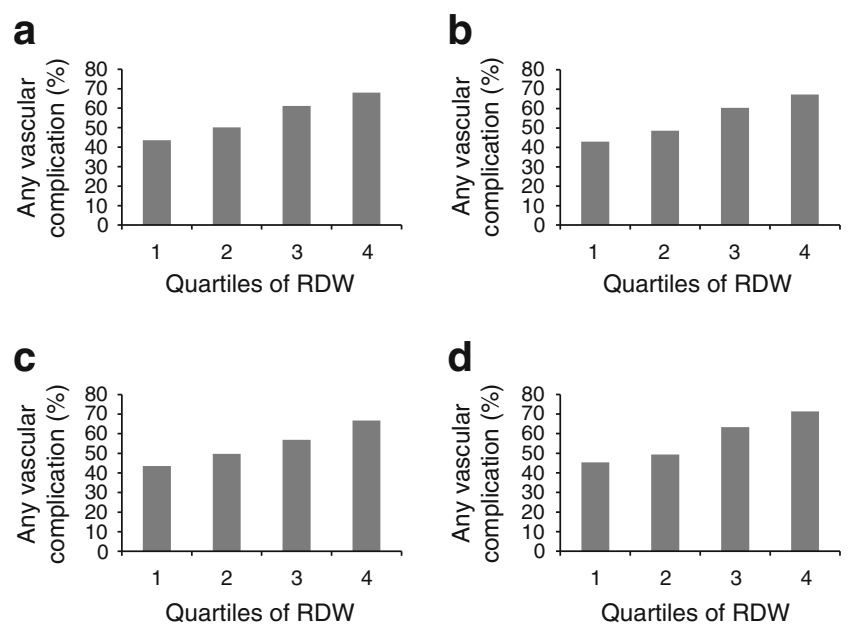

Fig. 1 Percentage of participants with at least one diabetic complication from the lowest to the highest quartile of RDW in the subgroup analyses. (a) The entire diabetic population $(p<0.001)$. (b) Diabetes participants excluding those recently treated for anaemia $(p<0.001)$,

(c) those with RDW values outside the normal range $(p<0.001)$ and

(d) those for whom vitamin $\mathrm{B}_{12}$ values were missing $(p<0.01)$ healthy participants who later developed type 2 diabetes. Likewise, increased concentrations of inflammatory markers, such as CRP and interleukin-6, have been involved in the development and progression of long-term diabetic macrovascular complications [18]. On the other hand, a shortened erythrocyte half-life has been reported in diabetes [19] and the metabolic syndrome has been related to increased RDW levels [20], leading us to postulate a possible influence of an underlying inflammatory state (the occurrence of which is typical in diabetes mellitus and the metabolic syndrome [18]) on accelerated erythrocyte destruction. In the present study, we observed increased CRP levels in the quartiles with highest RDW. In addition, the association between RDW and macrovascular disease was partially attenuated by adjustment for CRP. Thus, the relationship between RDW and CRP could explain, at least in part, our findings on the association between macrovascular disease and RDW in diabetes.

With regard to microvascular complications, we found an increased $\mathrm{OR}$ in the highest quartile of RDW for nephropathy, but not for retinopathy. These results could be explained by the presence of macrovascular and microvascular mechanisms in the pathogenesis of diabetic nephropathy [21], as opposed to mostly microvascular disease in diabetic retinopathy. In fact, atheromatous macrovascular alterations have been reported in diabetic patients with kidney disease $[21,22]$. On the other hand, our findings that the association between RDW and nephropathy was weaker than that with CVD may confirm the role of microvascular mechanisms as a major determinant of diabetic renal damage [21]. Studies enrolling individuals based on different inclusion/exclusion criteria to ours (i.e. general population, individuals with chronic heart failure) have recently reported a relationship between RDW and 
estimated GFR levels $<1 \mathrm{ml} \mathrm{s}^{-1} \mathrm{~m}^{-2}[23,24]$. Therefore, consistent with these studies [23, 24], we suggest that RDW also plays a role as a marker of nephropathy in patients with diabetes, possibly mediated through macrovascular damage.

The lack of association between RDW and retinopathy was unexpected. We had hypothesised that this microvascular complication would also be related to RDW levels, because of the proposed role of inflammation in the pathogenesis of retinopathy [18] and the association between retinopathy and an increased risk of cardiovascular morbidity and mortality [2]. Recent studies have reported that the relationship between diabetic retinopathy and systemic inflammatory markers, in particular CRP, is weak or non-existent [25-27]. Thus it is possible that microvascular disease and the related inflammatory process of retinopathy may occur mostly at the local tissue level and not be related to systemic inflammation $[18,26]$. Since RDW seems to reflect systemic inflammatory processes known to affect the macrovasculature [6, 24], this may explain, at least partially, its relationship with macrovascular but not microvascular disease processes.

Previous prospective studies have described a role of RDW as a predictive marker for CVD morbidity and mortality in several populations [4-7]. Also, in a recent meta-analysis, Patel et al. confirmed the relationship between RDW and increased risk of all-cause mortality in a subgroup of diabetic participants [28]. However, that meta-analysis was focused on elderly participants and did not investigate the specific relationship between RDW and vascular complications. Rather, Patel et al. investigated mortality in the overall population and in subgroups, including diabetic participants. We believe that our findings contribute to an understanding of the mechanisms relating RDW to mortality in diabetic participants.

Considering the cross-sectional nature of our study, our findings suggest that RDW plays a role as a predictive marker of macrovascular diabetic complications. If prospective studies confirm a predictive role of RDW, this would allow targeting of participants who might benefit from a different and more aggressive treatment of their diabetes and/or CVD risk factors, as well as from close screening and follow-up. For example, close monitoring of risk factors such as dyslipidaemia, hypertension and albuminuria might be recommended if increased RDW levels are present in diabetic patients, even without symptoms of CVD or with normal kidney function. RDW might help identify individuals for whom, at present, the decision to start treatment with anti-platelet agents for primary prevention of CVD is based on clinical judgment [1]. Also, RDW might play a role in recognising participants with increased risk of nephropathy before the appearance of albuminuria, thus identifying patients without evident nephropathy who might benefit from earlier treatment with drugs inhibiting the renin-angiotensinaldosterone system [29].

These results should be interpreted in light of the study's strengths and limitations. Its strengths include its large sample size and the wide assessment of coexistent macrovascular and microvascular risk factors in a random multiracial sample across the USA, providing excellent power and allowing adjustment for multiple covariates. However, there are also some important limitations. First, because of the cross-sectional design, we cannot establish causal relationships between RDW and diabetic complications. Second, CVD was defined through self-reported medical diagnosis, which could under- or overestimate the number of participants with macrovascular complications. The reliability of self-reported CVD is inconsistent in the literature, with results varying by patient population and study design. When comparing the medical records with patient self-report, some studies have found good agreement for CVD and stroke [30-33], while others have found lower agreement for the same conditions $[34,35]$. Nonetheless, it has been highlighted that structured interviews and questionnaires like those used in our study (i.e. NHANES III) increase the validity of collected data [31, $32,36]$. Third, considering that diabetic retinopathy was the outcome with the most missing data, our failure to find a relationship with RDW may result from lack of power; thus, larger studies will be useful to further evaluate the relationship between RDW and retinopathy. Fourth, although we considered several potential confounding factors in the regression models, the relationship between RDW and diabetic complications could still be influenced by unmeasured additional confounding factors. However, even if RDW is not part of the mechanistic pathway towards the development of vascular complications in diabetes, our findings still support the use of RDW as a clinical marker for the presence of vascular complications in diabetes.

In conclusion, this study demonstrates that higher RDW values are associated with increased CVD and nephropathy in a nationally representative sample of adults with diabetes in the USA. This association is independent of traditional risk factors, anaemia or treatment for anaemia, and duration of diabetes. The magnitude of the increased risk associated with higher RDW levels is comparable to other established markers such as CRP and $\mathrm{HbA}_{1 \mathrm{c}}$. Future prospective studies would be useful to provide additional information about the mechanisms relating RDW to macrovascular and microvascular disease, and the role of RDW as predictive marker of diabetic complications.

Duality of interest The authors declare that there is no duality of interest associated with this manuscript. 
Contribution statement NM and RJS participated in hypothesis formulation, study design, data analysis and interpretation, manuscript initial drafting and editing. WCW participated in study design, data analysis and interpretation, and manuscript editing. THT participated in data analysis and interpretation, and manuscript editing. HBW participated in study design, data interpretation and manuscript editing. All authors contributed to the final approval of the version to be published.

\section{References}

1. American Diabetes Association (2010) Standards of medical care in diabetes-2010. Diabetes Care 33(Suppl 1):11-61

2. Cheung N, Wong TY (2008) Diabetic retinopathy and systemic vascular complications. Prog Retin Eye Res 27:161-176

3. Evans TC, Jehle D (1991) The red blood cell distribution width. J Emerg Med 9(Suppl 1):71-74

4. Felker GM, Allen LA, Pocock SJ et al (2007) Red cell distribution width as a novel prognostic marker in heart failure: data from the CHARM Program and the Duke Databank. J Am Coll Cardiol 50:40-47

5. Tonelli M, Sacks F, Arnold M, Moye L, Davis B, Pfeffer M, for the Cholesterol and Recurrent Events (CARE) Trial Investigators (2008) Relation between red blood cell distribution width and cardiovascular event rate in people with coronary disease. Circulation 117:163-168

6. Patel KV, Ferrucci L, Ershler WB, Longo DL, Guralnik JM (2009) Red blood cell distribution width and the risk of death in middle-aged and older adults. Arch Intern Med 169:515-523

7. Perlstein TS, Weuve J, Pfeffer MA, Beckman JA (2009) Red blood cell distribution width and mortality risk in a communitybased prospective cohort. Arch Intern Med 169:588-594

8. No authors listed (1996) NHANES III Reports and Reference Manuals. National Center for Health Statistics, subsection Centers for Disease Control and Prevention. Available from www.cdc.gov/ nchs/nhanes/nh3rrm.htm\#manuals. Accessed 1 August 2010

9. No authors listed (1994) Plan and operation of the Third National Health and Nutrition Examination Survey, 1988-94: series 1. Vital Health Stat 1(32):1-407

10. Diabetic Retinopathy Study Group (1981) Report number 7. A modification of the Airlie House classification of diabetic retinopathy. Invest Ophthalmol Vis Sci 21:210-226

11. No authors listed (1996) NHANES III Examination Data File Documentation. National Center for Health Statistics, subsection Centers for Disease Control and Prevention. Available from www. cdc.gov/nchs/nhanes/nh3data.htm. Accessed 1 August 2010

12. Selvin E, Manzi J, Stevens LA et al (2007) Calibration of serum creatinine in the National Health and Nutrition Examination Surveys (NHANES) 1988-1994, 1999-2004. Am J Kidney Dis 50:918-926

13. Levey AS, Coresh J, Balk E et al (2003) National Kidney Foundation practice guidelines for chronic kidney disease: evaluation, classification, and stratification. Ann Intern Med 139:137-147

14. Looker AC, Dallman PR, Carroll MD, Gunter EW, Johnson CL (1997) Prevalence of iron deficiency in the United States. JAMA 277:973-976

15. Weiss G, Goodnough LT (2005) Anemia of chronic disease. N Engl J Med 352:1011-1023

16. Lippi G, Targher G, Montagnana M, Salvagno GL, Zoppini G, Guidi GC (2009) Relation between red blood cell distribution width and inflammatory biomarkers in a large cohort of unselected outpatients. Arch Pathol Lab Med 133:628-632

17. Libby P, Ridker PM, Maseri A (2002) Inflammation and atherosclerosis. Circulation 105:1135-1143
18. King GL (2008) The role of inflammatory cytokines in diabetes and its complications. J Periodontol 79(8 Suppl):1527-1534

19. Acosta J, Hettinga J, Flückiger R et al (2000) Molecular basis for a link between complement and the vascular complications of diabetes. Proc Natl Acad Sci USA 97:5450-5455

20. Sánchez-Chaparro MA, Calvo-Bonacho E, González-Quintela A et al (2010) Higher red blood cell distribution width is associated with the metabolic syndrome: results of the Ibermutuamur Cardiovascular Risk Assessment study. Diabetes Care 33:e40

21. Zuccalà A, Losinno F, Zuchelli A, Zuchelli PC (1998) Renovascular disease in diabetes mellitus: treatment by percutaneous transluminal renal angioplasty. Nephrol Dial Transplant 13:26-29

22. Sawicki PT, Kaiser S, Heinemann L, Frenzel H, Berger M (1991) Prevalence of renal artery stenosis in diabetes mellitus-an autopsy study. J Intern Med 229:489-492

23. Lippi G, Targher G, Montagnana M, Salvagno GL, Zoppini G, Guidi GC (2008) Relationship between red blood cell distribution width and kidney function tests in a large cohort of unselected outpatients. Scand J Clin Lab Invest 68:745-748

24. Förhécz Z, Gombos T, Borgulya G, Pozsonyi Z, Prohászka Z, Jánoskuti L (2009) Red cell distribution width in heart failure: prediction of clinical events and relationship with markers of ineffective erythropoiesis, inflammation, renal function, and nutritional state. Am Heart J 158:659-666

25. Izuora KE, Chase HP, Jackson WE et al (2005) Inflammatory markers and diabetic retinopathy in type 1 diabetes. Diabetes Care 28:714-715

26. Meleth AD, Agrón E, Chan CC et al (2005) Serum inflammatory markers in diabetic retinopathy. Invest Ophthalmol Vis Sci 46:4295-4301

27. Spijkerman AM, Gall MA, Tarnow L et al (2007) Endothelial dysfunction and low-grade inflammation and the progression of retinopathy in type 2 diabetes. Diabet Med 24:969-976

28. Patel KV, Semba RD, Ferrucci L et al (2010) Red cell distribution width and mortality in older adults: a meta-analysis. J Gerontol A Biol Sci Med Sci 65:258-265

29. Ruggenenti P, Cravedi P, Remuzzi G, Medscape (2010) The RAAS in the pathogenesis and treatment of diabetic nephropathy. Nat Rev Nephrol 6:319-330

30. Bush TL, Miller SR, Golden AL, Hale WE (1989) Self-report and medical record report agreement of selected medical conditions in the elderly. Am J Public Health 79:1554-1556

31. Bergmann MM, Byers T, Freedman DS, Mokdad A (1998) Validity of self-reported diagnoses leading to hospitalization: a comparison of self-reports with hospital records in a prospective study of American adults. Am J Epidemiol 147:969-977

32. Lampe FC, Walker M, Lennon LT, Whincup PH, Ebrahim S (1999) Validity of a self reported history of doctor-diagnosed angina. J Clin Epidemiol 52:73-81

33. Tretli S, Lund-Larsen PG, Foss OP (1982) Reliability of questionnaire information on cardiovascular disease and diabetes: cardiovascular disease study in Finnmark county. J Epidemiol Community Health 36:269-273

34. Klungel OH, de Boer A, Paes AH, Seidell JC, Bakker A (1999) Cardiovascular diseases and risk factors in a population-based study in the Netherlands: agreement between questionnaire information and medical records. Neth J Med 55:177-183

35. O'Donnell CJ, Glynn RJ, Field TS et al (1999) Misclassification and under-reporting of acute myocardial infarction by elderly persons: implications for community-based observational studies and clinical trials. J Clin Epidemiol 52:745-751

36. Marquis KH, Cannell CF (1971) Effect of some experimental interviewing techniques on reporting in the health interview survey. Vital Health Stat 2, no. 41. Available from www.cdc. gov/nchs/products/series/series02.htm. Accessed 15 April 2011 